\title{
The development of eyespot patterns on butterfly wings: morphogen
}

\section{sources or sinks?}

\author{
VERNON FRENCH ${ }^{1}$ and PAUL M. BRAKEFIELD ${ }^{2}$ \\ ${ }^{1}$ Institute of Cell, Animal and Population Biology, University of Edinburgh, Kings Buildings, West Mains Road, Edinburgh EH9 3JT, UK \\ ${ }^{2}$ Section of Evolutionary Biology, Department of Population Biology, University of Leiden, Schelpenkade 14A, 2313 ZT Leiden, The Netherlands
}

\section{Summary}

We have studied the development of the eyespot colour pattern on the adult dorsal forewing of the nymphalid butterflies, Bicyclus safitza and B. anynana, by cauterising the presumptive eyespot centres (the foci) on the pupal wing. The effects on pattern depended on age at cautery. Early focal cautery (at 1-12 hours after pupation) usually reduced or eliminated the eyespot, while cautery at a non-focal site usually had no effect. These results resemble those of a previous study on another species but, in addition, we find that a later cautery (at 12-24 hours) had the converse effect of generating pattern, so that focal cautery enlarged the anterior eyespot (but usually not the large posterior eyespot) and nonfocal cautery induced a new ectopic eyespot.

The effects of cautery on patterning are more extensive by an order of magnitude than the cell death which is caused, so implicating a long-range mechanism, such as a morphogen gradient, in eyespot development. The focus clearly acts to establish the normal eyespot pattern, but a simple source/diffusion model is not supported by the response to late cautery. We suggest two alternative forms of gradient model in which late damage can mimic and augment the action of a focus. In the Source/Threshold model, the focus is a morphogen source, and cautery can remove the focus but also transiently lowers the response threshold in surrounding cells. In the Sink model, the focus generates the gradient by removing morphogen, and cautery can eliminate the focus but it also causes a transient destruction or leakage of morphogen. These models can explain most features of the results of cautery.

Key words: eyespot, butterfly wing, pattern formation, gradient.

\section{Introduction}

The spectacular colour patterns on butterfly and moth wings are among the most diverse products of biological pattern formation, but they can be understood as variations on a theme which consists of an array of simple elements such as bands and eyespots (Schwanwitsch, 1924; Suffert, 1927; Nijhout, 1978, 1991). The adult wing is a mosaic of coloured scales whose spatial pattern is specified in the larval and early pupal epidermis, long before scales are formed or pigment is synthesised (Nijhout, 1991).

An eyespot is a set of concentric rings of colour which usually occurs in the distal part of the wing, centred midway between adjacent wing veins. Nijhout (1980) showed that the dorsal forewing eyespot of the nymphalid butterfly, Precis coenia, was reduced in size by microcautery of its presumptive centre (the 'focus') in the early pupa. Furthermore, grafting the focus to a different position caused a small eyespot pattern to form in the surrounding epidermis (Nijhout, 1980). Clearly, the eyespot is specified by a signal from the focus, and Nijhout $(1978,1980)$ has proposed that this signal is an unstable molecule (a 'morphogen') which is produced at the focus and diffuses away to form a radial concentration gradient. If morphogen concentration determined the colour of scales formed by the epidermal cells, the gradient levels would define the rings of an eyespot pattern. The adjacent gradients from a row of foci would merge to form a morphogen ridge whose levels could define bands across the adult wing (Nijhout, 1978, 1985a, 1990; Bard and French, 1984). However, cautery of the hindwing of Precis induced the formation of an eyespot-like pattern (Nijhout, 1985a). This ectopic response is not readily explained by the simple gradient model, and it is difficult to relate it to the response to cautery of a normal eyespot, as they occur on different wing surfaces in Precis.

In the present work on Bicyclus butterflies we, like Nijhout (1980), find that an early focal cautery reduces (or completely eliminates) the eyespot but, at a later stage, eyespot size is substantially increased, and a non-focal cautery can induce an ectopic eyespot. These results have considerable implications. They support a long-range patterning mechanism (such as a morphogen gradient), but indicate that a simple source/diffusion model is inadequate to explain eyespot development. 


\section{Materials and methods}

\section{Experimental animals}

Operations were performed on pupae of Bicyclus safitza and B. anynana (Order, Lepidoptera; Family, Nymphalidae; Sub-family, Satyrinae) from laboratory stocks descended from gravid females captured in Malawi. Stocks were maintained at $26 \pm 1^{\circ} \mathrm{C}$, with high humidity and a 12:12 hour photoperiod; the larvae were fed on maize plants and adults on mashed banana. Larvae reared in these conditions develop into the 'wet season' form of butterfly, with full eyespot patterns (Brakefield and Reitsma, 1991). Final instar larvae stop feeding and form immobile prepupae about 24 hours before pupation, which usually occurs within a few hours of the onset of darkness. The prepupae were collected and checked regularly so that pupation times were usually known to within \pm 10 or 15 minutes.

\section{Microcautery and analysis}

Cautery experiments were performed on the pupal left wing, using an electrolytically-sharpened tungsten needle attached to the heating element of a variable power source. The cuticle and underlying epidermis of the dorsal forewing were pierced at sites identified by the wing veins and cuticular marks (see Fig. 1D). Pupae were cauterised at various ages ( 1 hour, 6 hours, etc) and then returned to $26^{\circ} \mathrm{C}$ until eclosion (7-8 days). The adults were killed after wing expansion, both forewings then removed, and the eyespots drawn with a camera lucida and their areas measured using a DIFA image analysis system (Windig, 1992). In untreated butterflies, the left and right forewings are very similar (eyespot area ratios were $0.85-1.15$ in $95 \%$ of animals), but there was considerable variability among individuals; the experimental pattern was therefore always related to its contralateral control wing. Area ratios of the experimental-to-control eyespot were calculated and analysed using the MINITAB statistical package.

\section{Severity of cautery}

Cautery was standardised by controlling the current, the profile of the needle, the depth to which it was inserted and the time for which it was left in place. In the main experiment, cautery was done at several severities (from an unheated needle withdrawn immediately, to a $70^{\circ} \mathrm{C}$ needle tip inserted for 4 seconds), but there was little evidence of an effect of severity of cautery on the result. 2-way Analysis of Variance showed no significant major effect of severity $(F=2.38$; df $=3,283 ; P=\mathrm{n} . \mathrm{s}$, for most severities over all time points in B. safitza; $F=1.06$; $\mathrm{df}=1,188 ; P=\mathrm{n} . \mathrm{s}$, for the $B$. anynana data), and little indication of a systematic effect, so the data to be analysed were pooled with respect to severity. However, further analysis for all severities showed a systematic effect at 6 hours in $B$. safitza $(F=3.12$; df $=4,52 ; P<0.05)$, and this was explored with a separate 6 hour severe cautery experiment on both species.

\section{Damage caused by cautery}

For examination of the extent of cell death, some pupal wings were removed shortly after a 1-hour or 18-hour cautery, dissected, fixed and stained with basic fuchsin. An unheated needle damaged the dorsal epidermis, causing an area of dead cells of around $125 \mu \mathrm{m}$ in diameter, which corresponds to approximately 220 cells at 1 hour ( 460 cells at 18 hours, after pupal cell divisions have occurred). Damage was variable and more extensive after a more severe cautery.

An operation locally disturbed the arrangement of adult scales, and the site of cautery was often occupied by light-brown scales which may correspond to the normal ground scales (Figs 1C, 3E).
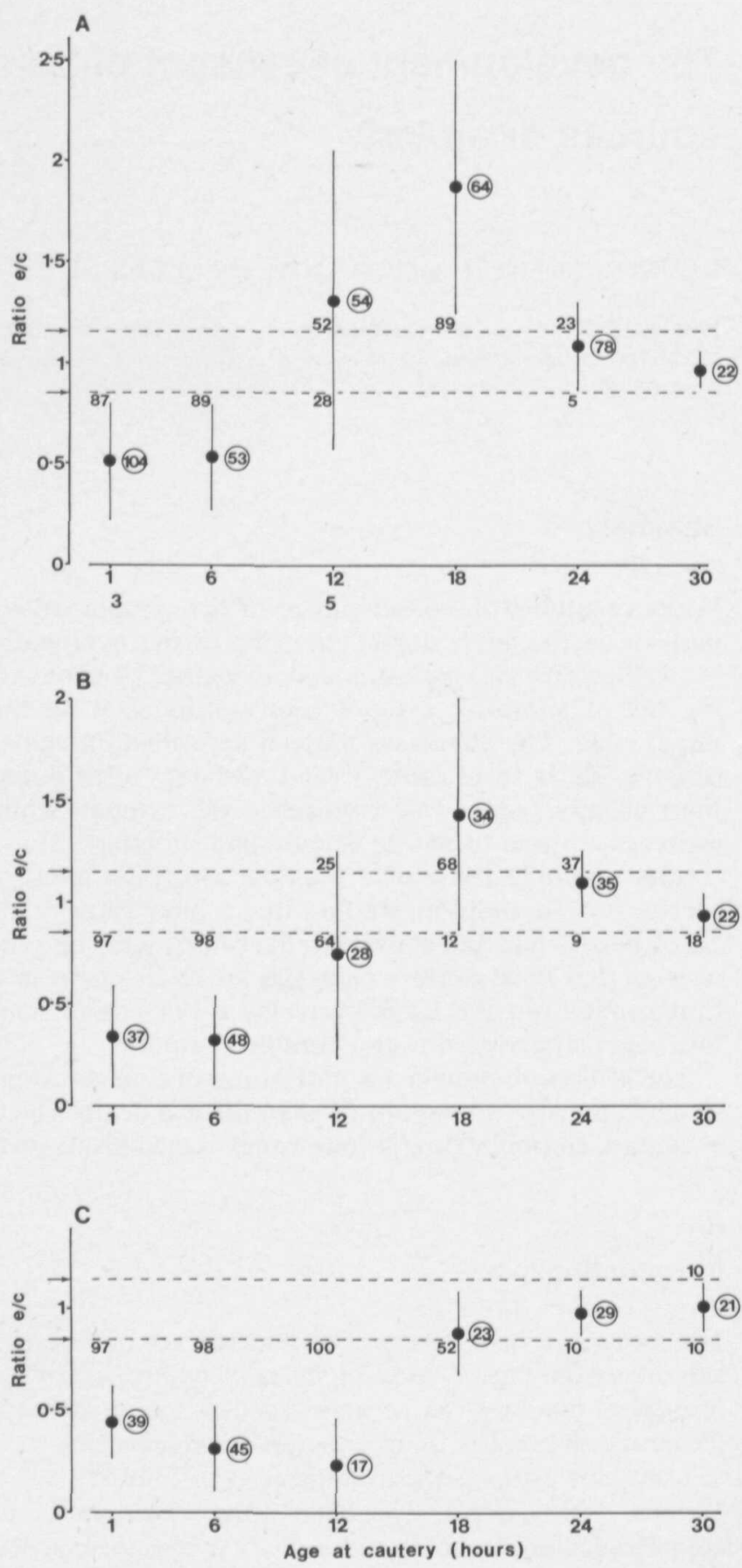

In the severe 6-hour cautery experiments, damage was much more pronounced, with the wing surface often crumpled, the vein pattern distorted and a scale-less area around the site of cautery.

\section{Results}

The dorsal forewing bears a small anterior and a large posterior eyespot (Fig. 1). The development of this pattern was studied by cauterising pupae of various ages (1-30 hours after pupation) at the presumptive centre (the focus) of the anterior and, in B. anynana, of the posterior eyespot, and also more distally in an adjacent wing-cell (Fig. 1D). Cautery caused dramatic alterations in the eyespot patterns 
Fig. 2. Effects of focal cautery on size of the anterior (A,B) and posterior (C) eyespot. The ratio of total areas of the cauterised experimental and contralateral control eyespots (e/c) is shown (as the mean with standard deviation) following cautery at various times after pupation. The figures at each time point are the percentages (over $5 \%$ ) of animals showing a decrease or an increase in eyespot size (i.e. an e/c ratio below 0.85 or above 1.15 , respectively). Small figures in circles are the number of animals with measurable cauterised and control eyespots. (A) Cautery of the anterior focus in B. safitza. The decreases in total eyespot size caused at 1 hour and 6 hours were not significantly different (Mann-Whitney test $-W=6075$; d.f. $=90,47 ; P=$ n.s.), whereas those at 12 hours were less extreme $(W=1337$; d.f. $=47,15 ; P=$ $0.019)$. The increases in total eyespot size caused at 18 hours were more extreme than those at 12 hours $(W=983 ;$ d.f. $=28,57 ; P=$ $0.04)$ or at 24 hours $(W=2537$; d.f. $=57,18 ; P<0.01)$. (Analysis of the area of the dark-brown regions of the eyespots gave very similar results.) (B) Cautery of the anterior focus in B. anynana. Decreases in eyespot size were equally severe at 1 hour or 6 hours, less extreme at 12 hours and even less extreme in the few cases induced at 18 hours ( differences were not quite statistically significant). Increases in total size at 12 hours and 18 hours were similar, and more extreme than those caused later, at 24 hours ( $W$ $=541$; d.f. $=30,13 ; P<0.001$ ). (C) Cautery of the posterior focus in B. anynana. Decreases in posterior eyespot total size were less extreme at 1 hour than later, at 6 hours $(W=1937$; d.f. $=38,44 ; P$ $<0.001)$ or at 12 hours. The decreases at 12 hours were also more extreme than those caused later, at 18 hours $(W=164$; d.f. $=$ 17,12; $P<0.001$ ) or 24 hours. (Analysis of the dark-brown regions gave very similar results, with a maximum decrease following cautery at 6 and 12 hours.)

and in the adjacent region, with these effects depending critically on the age at cautery (see below).

\section{Microcautery of the anterior focus}

Microcautery of the focus of the anterior eyespot in the early pupa (at 1-12 hours) usually caused a decrease in the size of the eyespot on the adult wing. In both species of Bicyclus, however, a slightly later operation (at 12-24 hours) often caused a major increase in eyespot size (Fig. 2A,B).

In extreme cases, early microcautery entirely eliminated the anterior eyespot (Fig. 3A) or reduced it to a patch of gold scales (Fig. 3C). Usually a small but complete eyespot developed, with some central white scales (often associated with light-brown ground scales) surrounded by darkbrown and gold annuli (Fig. 3B), and the borders were less clearly defined than in a control pattern. Large decreases in eyespot size were caused at high frequency by microcautery at 1 hour or at 6 hours, but the decreases became both less frequent and less severe following later operations, at 12 hours (see Fig. 2A,B).

The enlarged eyespots which formed following later cautery showed similar size increases in the dark-brown and gold regions, but not in the central spot of white scales (which was usually reduced and often surrounded by the ground scales - Fig. 3D). As in the reduced eyespots, borders between the regions of the enlarged eyespots were usually rather vague. The increases in eyespot size occurred from 12 to 24 hours, but were both most frequent and most extreme following operations in the middle of the period, at 18 hours (see Fig. 2A,B).

\section{Microcautery of the posterior focus}

Posterior focal cautery was performed only on B. anynana. Decreases in the size of the large eyespot were caused at high frequency from 1 hour to 18 hours (Fig. 2C), and the resulting patterns ranged from elimination to a small but complete eyespot (Fig. 3C), with the most severe effects at 12 hours after pupation. Increases in posterior eyespot size were very slight and occurred only rarely, at 18-30 hours (Fig. 2C). At an individual level, effects on the anterior and posterior eyespots were correlated (eg. at 18 hours the Spearman-Rank correlation between eyespot areas, both relative to their controls, was 0.57 - decreases in both eyespots were thus associated, as were increases in the anterior and no change in the posterior eyespot).

\section{Non-focal microcautery - ectopic eyespots}

Microcautery at the distal site (Fig. 1D) frequently caused the formation of an ectopic pattern: this ranged from a few gold scales, to a gold patch, to an ectopic eyespot consisting of dark-brown and surrounding gold scales (Fig. 3D). At the site of cautery, there were usually light-brown ground scales, but the white scales which form the centre of a normal eyespot never appeared.

The effect of non-focal cautery clearly depended on age. In $B$. safitza, a full ectopic eyespot was induced at high frequency only at 12-21 hours (the period when the anterior eyespot could be enlarged by focal cautery), and there was usually no effect at 1 hour or at 24 hours or later (see Fig. 4). The ectopic eyespots were largest when induced at 12 hours. At an individual level, the effects of the focal and

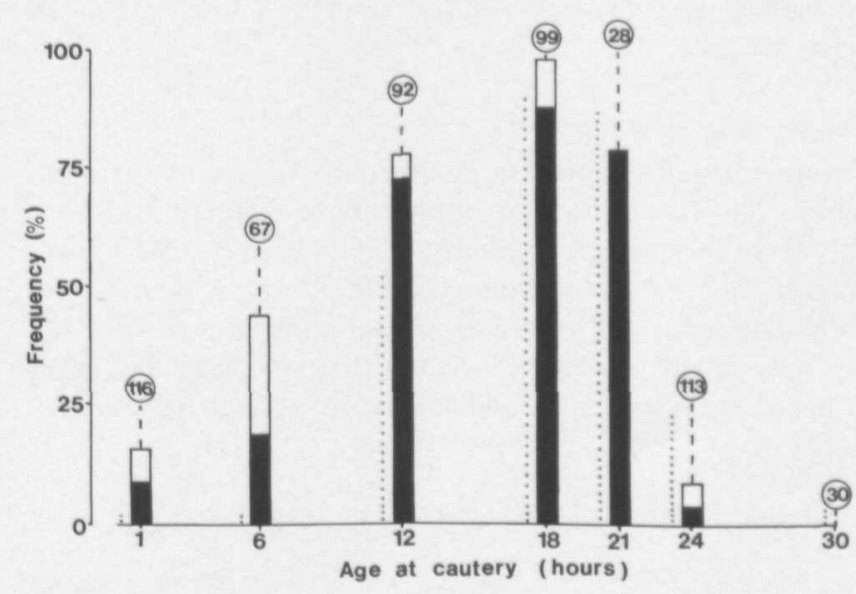

Fig. 4. Induction of ectopic pattern by non-focal cautery in $B$. safitza. For each time point, the frequency (\%) is given for ectopic eyespots (filled bar), gold patches (open bar) and for scattered gold scales (dashed line). Above each bar is the total number of scorable patterns, and the dotted line indicates the frequency of enlarged anterior eyespots resulting from focal cautery at that time. Ectopic eyespots induced at 12 hours were larger than those at 6 hours $(W=163$; d.f. $=59,19 ; P<0.001)$ or at 18 hours $(W=$ 2130 ; d.f. $=59,80 ; P=0.01$ ). Ectopics induced at 18 hours were larger than at 21 hours (from analysis of dark-brown regions - $W=$ 3634 ; d.f. $=18,80 ; P<0.001)$. Note: the total number of animals at each time point exceeds that in Fig. $2 \mathrm{~A}$ because some were scorable for ectopic pattern but not measurable for anterior eyespot size. 
non-focal cauteries were strongly correlated, as animals with large increases in the anterior eyespot tended to have large ectopics, and those with no increase or a decrease had small ectopics (Spearman-Rank correlation between the areas of cauterised anterior and ectopic eyespots, both relative to the control anterior eyespot, was 0.78 ).

In $130 / 142$ B. safitza adults, the cauterised anterior eyespot was larger than the ectopic (mean area ratio $=1.59$ ). Similarly, when areas of the inner dark-brown regions of the cauterised anterior and the ectopic eyespot were compared, the former was almost always larger (149/162 cases - mean ratio $=1.39$ ). Some $B$. anynana pupae were also cauterised at the non-focal site, and the results (not shown) resembled those of $B$. safitza, with the cauterised anterior eyespot again consistently larger than the ectopic (mean area ratio $=2.38$ ). Hence cautery in the position of a normal focus consistently produced a larger eyespot pattern, in both species, than a similar cautery could induce at another site: normal pattern formation and the effect of the cautery are (to some degree) additive.

\section{Effects of cautery on the ventral pattern}

The ventral wing surface also has two eyespots, which are directly beneath the dorsal ones, and this ventral pattern could be altered by microcautery at 1-12 hours. In B. anynana, there were frequent major reductions in the large posterior eyespot, but in other ways the ventral response differed from that found dorsally. The increases and decreases in anterior eyespot size were slight and occurred only at low frequency $(5-10 \%)$; at the non-focal site, gold scales (or a small ectopic eyespot) occurred only infrequently, and no ventral effects were seen in animals cauterised at 18 hours or later.

\section{Severe cautery at 6 hours}

Severe cautery resulted in distortion of many of the adult wings, and the effects on wing pattern differed considerably from those of the milder cautery in both $B$. safitza (data not shown) and $B$. anynana (Table 1). At 6 hours, mild focal cautery usually reduced dorsal eyespot size (Fig. 2), whereas severe cautery produced frequent large increases in anterior eyespot size, and even a few slight increases in the large posterior eyespot of B. anynana (Fig. 5A). Severe non-focal cautery induced an ectopic eyespot in most of the butterflies (Table 1). In both species, the dorsal effects of severe 6-hour cautery were very similar to those found later, at 12 or 18 hours, with milder cautery. Ventrally, the severe 6 -hour cautery produced frequent reductions in the eyespots (which were difficult to assess due to extensive damage), and frequent gold patches or small ectopic eyespots at the non-focal site (Table 1, Fig. 5B).

\section{Discussion}

Our results on Bicyclus safitza and B. anynana, like those of Nijhout (1980) on Precis coenia, show that early focal cautery can decrease the size of an eyespot, reduce it to the peripheral gold scales or completely eliminate it. In Bicyclus, moreover, a slightly later operation can induce the epidermis to generate pattern around the area of damage, increasing the size of the small anterior (and, occasionally, of the large posterior) eyespot, and producing a new pattern at a non-focal site (Figs 2,3). There was a clear effect of age on the response to cautery but, at any one time point, the results were rather variable (see Fig. 2). Much of this variability may derive from individual differences in precise developmental stage, as indicated by the high correlations, at an individual level, between the results of cautery at the anterior focus, at the non-focal site and, in B. anynana, at the posterior focus .

Bicyclus ectopic patterns ranged from a few gold scales to an eyespot larger than the control anterior eyespot, but the centre was occupied by light-brown ground scales and never by the white scales found in normal eyespots. In addition, the white centre was never increased in an enlarged eyespot (eg. Fig. 3D). These results indicate that the central cells were already determined in the larva, not only to act as the focus for eyespot formation, but also to form the white adult scales. An ectopic eyespot fused smoothly with an adjacent cauterised (Figs 3D, 5A) or normal eyespot, suggesting that the late cautery and the normal focus generate pattern by the same mechanism. Furthermore, in both Bicyclus species, the cauterised anterior eyespot was almost always larger than an ectopic induced

Table 1. Effect of mild and severe cautery of the 6-hour pupal wing on the pattern on both wing surfaces of Bicyclus anynana

\begin{tabular}{|c|c|c|c|c|c|c|c|c|c|c|c|c|}
\hline \multirow{3}{*}{$\begin{array}{l}\text { Severity } \\
\text { of } \\
\text { cautery }\end{array}$} & \multicolumn{8}{|c|}{ Focal cautery } & \multirow{2}{*}{\multicolumn{4}{|c|}{$\begin{array}{l}\text { Non-focal cautery } \\
\text { induced pattern }\end{array}$}} \\
\hline & \multicolumn{4}{|c|}{ Anterior eyespot } & \multicolumn{4}{|c|}{ Posterior eyespot } & & & & \\
\hline & $N$ & decr & NE & incr & $N$ & decr & NE & incr & $N$ & NE & gold & eyespot \\
\hline \multicolumn{13}{|l|}{ (i) Dorsal } \\
\hline Mild & 48 & 98 & 2 & - & 45 & 98 & 2 & - & 29 & 79 & 21 & - \\
\hline Severe & 22 & 32 & 18 & 50 & 22 & 86 & 5 & 9 & 23 & - & - & 100 \\
\hline \multicolumn{13}{|l|}{ (ii) Ventral } \\
\hline Mild & 52 & 4 & 94 & 2 & 50 & 64 & 36 & - & 23 & 100 & - & - \\
\hline Severe & 23 & 100 & - & - & 23 & 100 & - & - & 23 & 57 & 30 & 13 \\
\hline
\end{tabular}

The table gives the number of scorable animals $(N)$; the percentages with a decrease (decr), no effect (NE) and an increase (incr) in eyespot size after focal cautery; and with no pattern (NE), gold scales or a gold patch (gold), and an eyespot at the site of non-focal cautery. Results occurring at much higher frequency after severe cautery are given in bold type. Because of wing distortion following severe cautery, eyespot areas could not be measured accurately. 

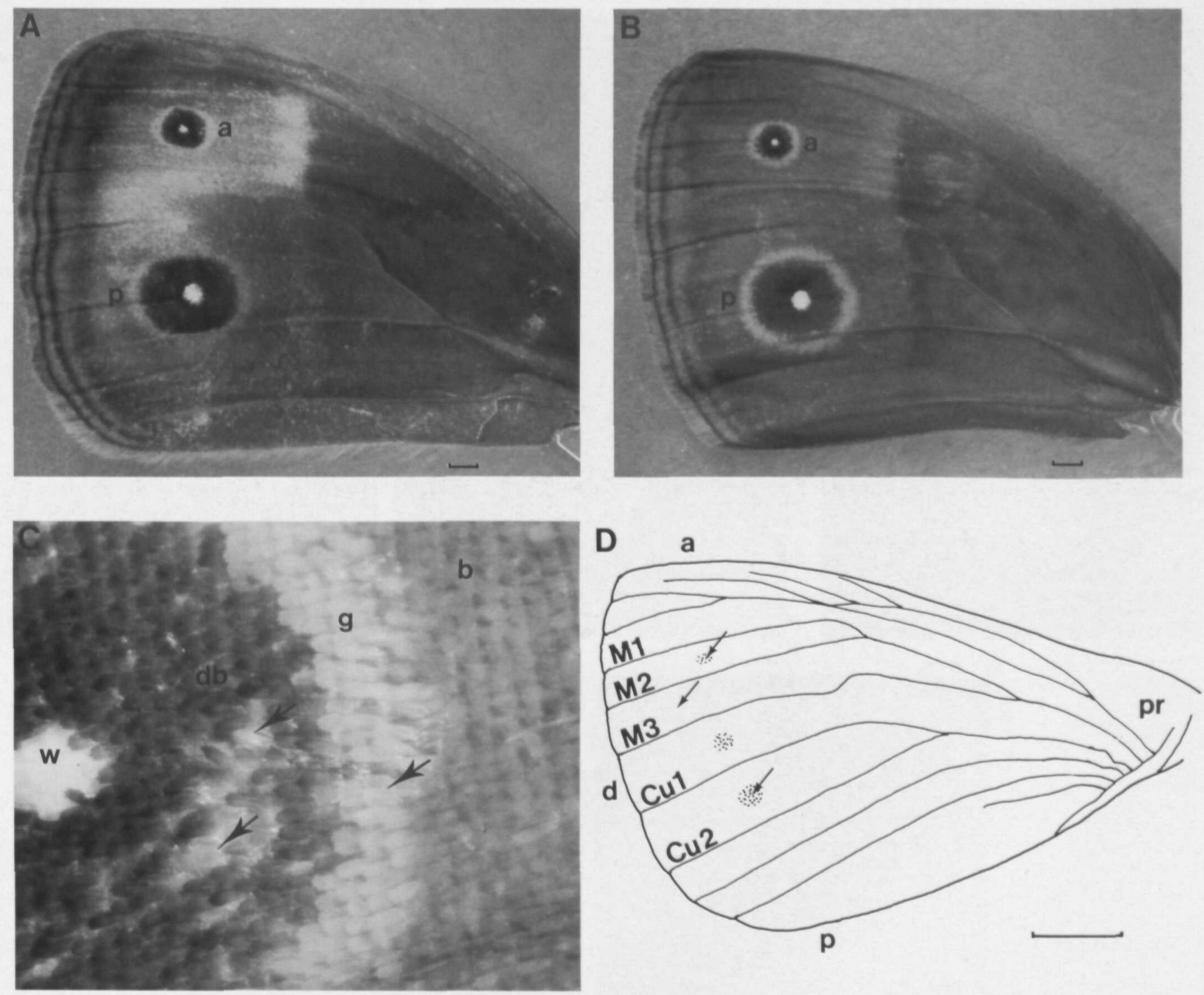

Fig. 1. Adult and pupal wings of Bicyclus. (A,B) Dorsal surface of the left forewings of adults of (A) B. safitza and (B) B. anynana. The small anterior (a) and large posterior (p) eyespots each have a white centre surrounded by dark-brown and a peripheral gold annulus. The background is mostly light- or mid-brown, but in $B$. safitza $(\mathrm{A})$ there is a diffuse gold area proximal and posterior to the anterior eyespot. In both species, occasional butterflies also have one or more small eyespots in other positions. Scale bar - $1 \mathrm{~mm}$. (C) The wing surface of B. anynana, showing the white scales $(\mathrm{w})$ at the centre of the anterior eyespot, the surrounding dark-brown $(\mathrm{db})$ and gold $(\mathrm{g})$ scales, and the mid-brown (b) scales on the general wing surface. Within each transverse row there is an approximate alternation of long 'cover scales' and underlying light-brown 'ground scales', and this specimen has been rubbed slightly to expose the ground scales (arrows) in some places. (D) Camera lucida drawing of the pupal forewing of B. anynana, showing the vein pattern (see Nijhout, 1985b), and the cuticular marks (stippling) at the presumptive centres of the eyespots (and in one adjacent position). a, anterior; p, posterior; pr, proximal; $\mathrm{d}$, distal. Arrows indicate the sites of cautery; in $B$. safitza, the posterior focus was not used, as the large posterior eyespot is often poorly defined. Scale bar, $1 \mathrm{~mm}$. 

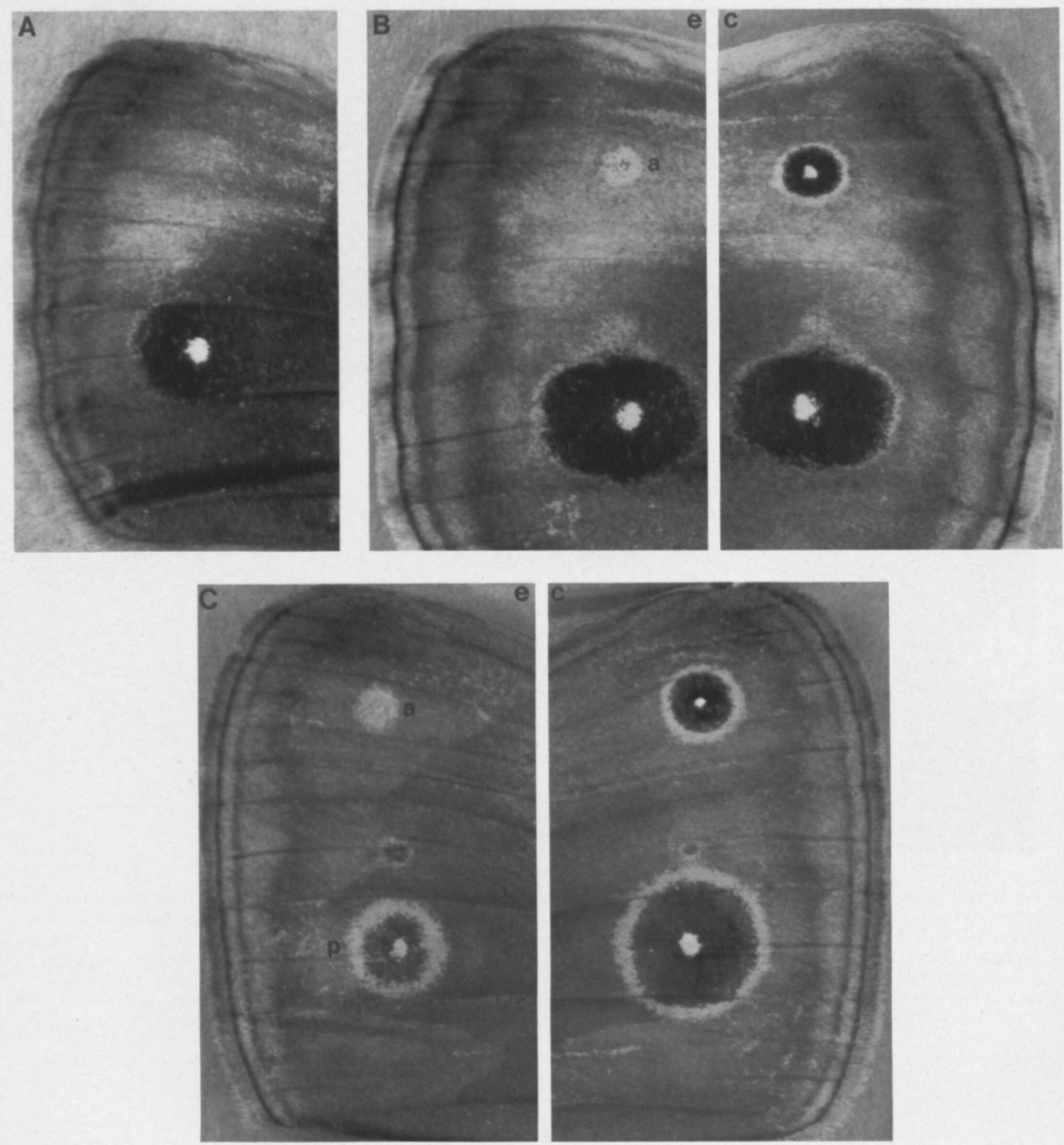

. 

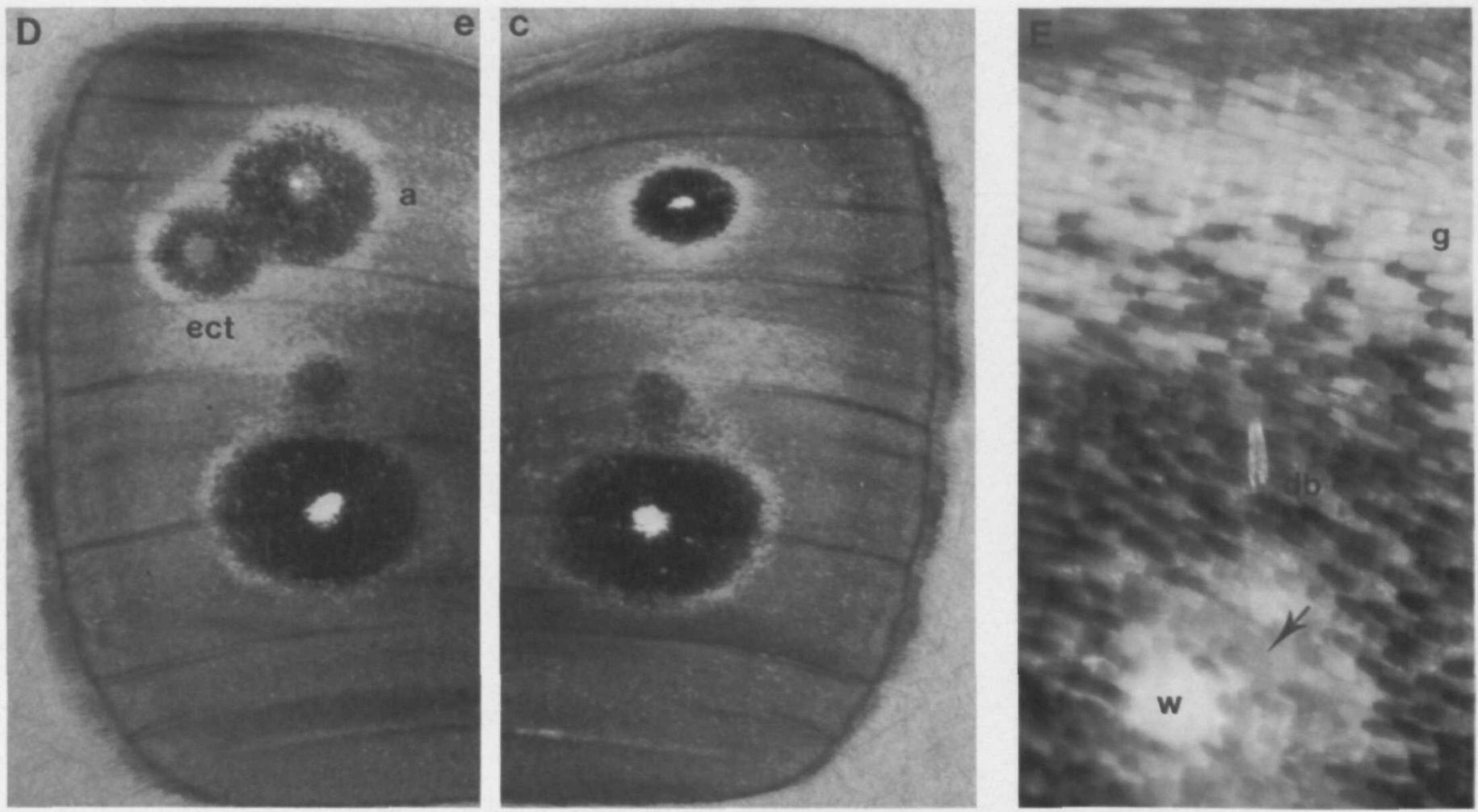

Fig. 3. Effects of focal and non-focal cautery on the dorsal forewing pattern. (A) Experimental wing of $B$. safitza cauterised at 6 hours, showing elimination of the anterior eyespot, and no ectopic pattern at the site of non-focal cautery. (B) The experimental (e) and control (c) wings of B. safitza cauterised at 1 hour, showing a great decrease in size of the anterior eyespot (a). (C) The experimental (e) and control (c) wings of B. anynana cauterised at 6 hours, showing the anterior (a) eyespot reduced to a patch of gold scales, the posterior eyespot (p) decreased in size, and no response to non-focal cautery. (D) Experimental (e) and control (c) wings of $B$. safitza cauterised at 18 hours, showing enlargement of the anterior eyespot and a large ectopic eyespot (ect) caused by non-focal cautery. (E) Magnification of part of the cauterised anterior eyespot from (D), showing the central region of white scales (w) and ground scales (arrow), and part of the enlarged dark-brown $(\mathrm{db})$ and gold $(\mathrm{g})$ regions. 

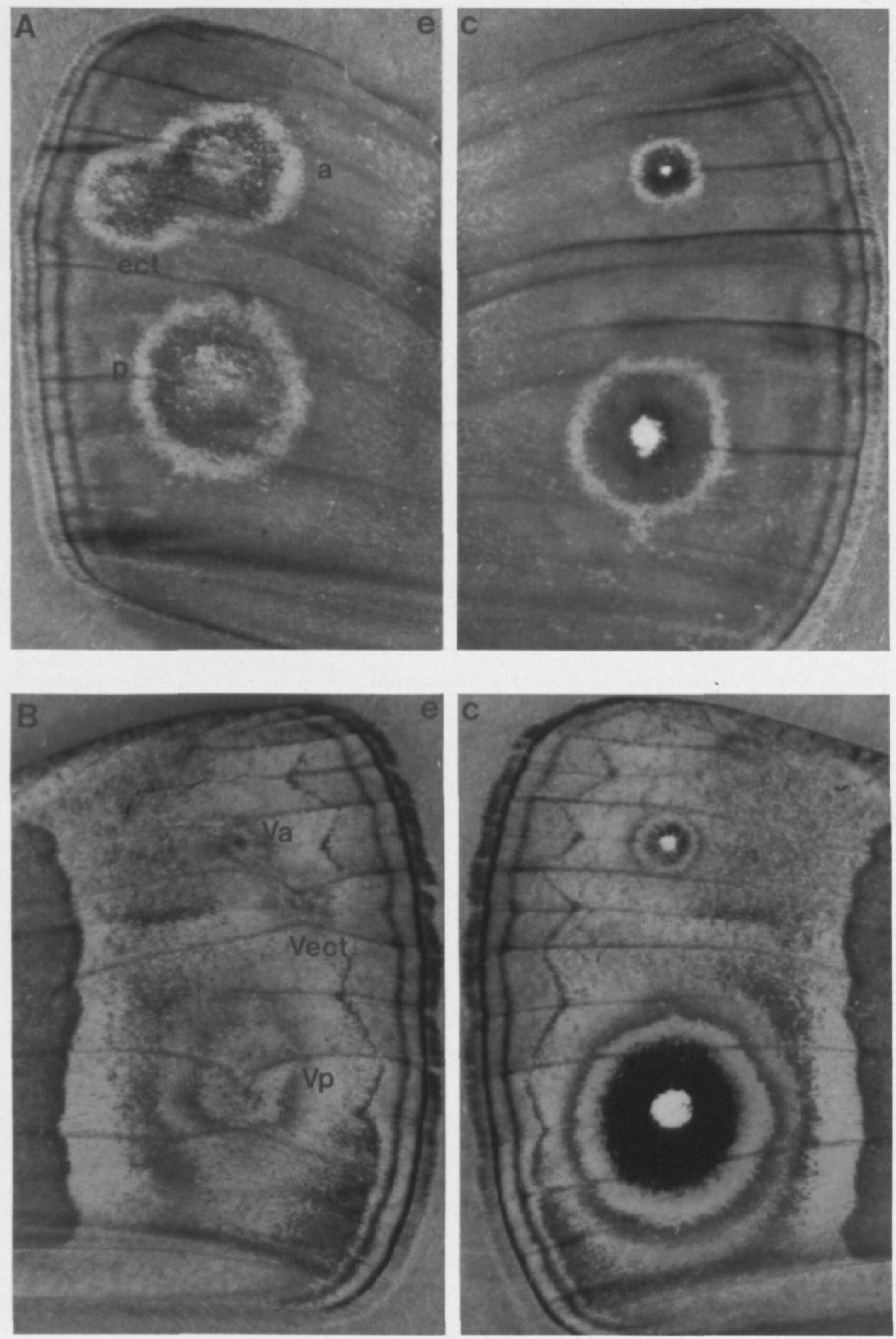

Fig. 5. Effect of severe focal and non-focal 6-hour cautery on the forewing of B. anynana. (A) The dorsal surfaces of experimental (e) and control (c) wings, showing a large increase in size of the anterior eyespot (a), a slight increase in the posterior eyespot (p), and a large ectopic eyespot (ect) at the site of non-focal cautery. (B) The ventral surfaces of experimental (e) and control (c) wings, showing great reductions in anterior $(\mathrm{Va})$ and posterior $(\mathrm{Vp})$ eyespots, and the formation of a small ectopic eyespot $(\mathrm{V}$ ect) at the non-focal site. The damage caused by the severe cautery is evident on both wing surfaces. 
A
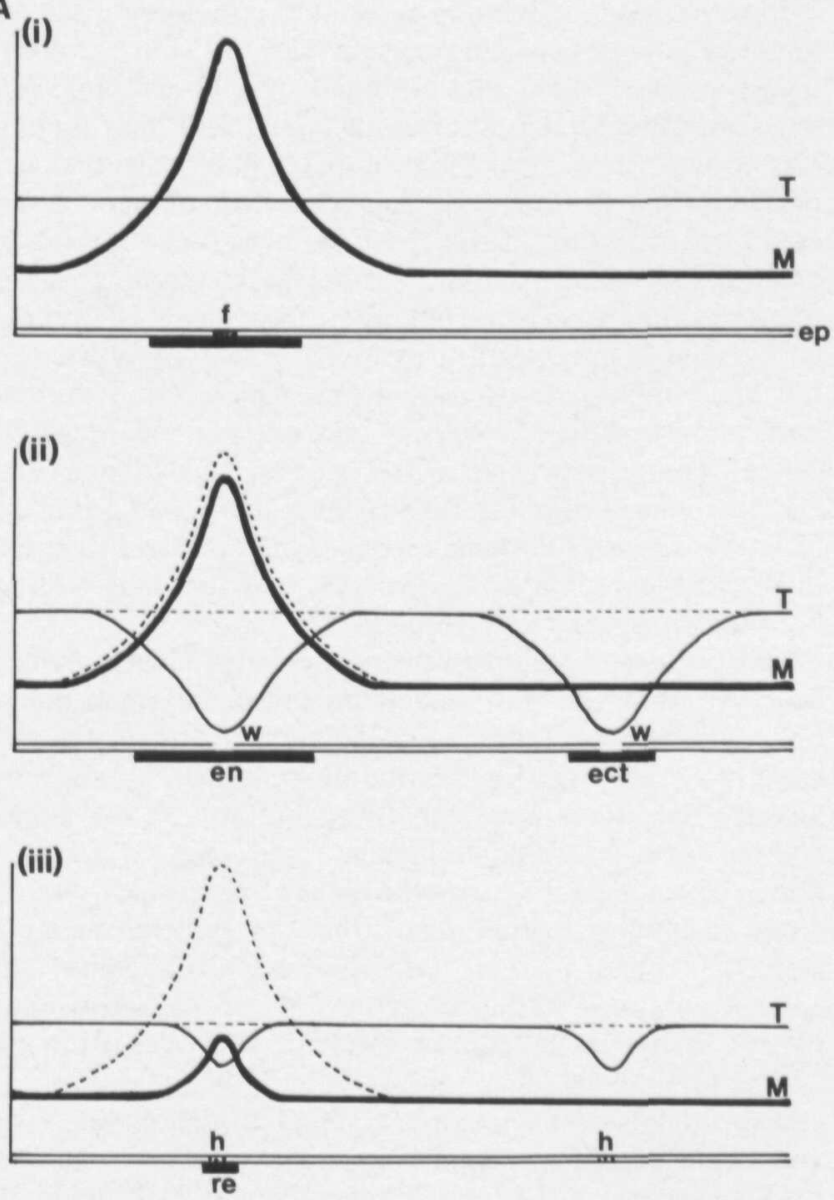

B (i)

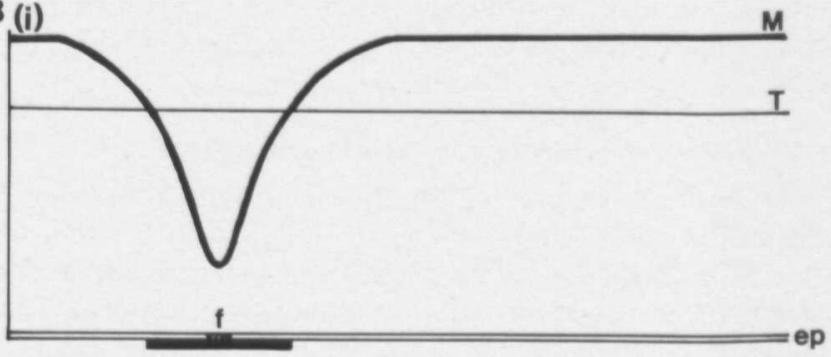

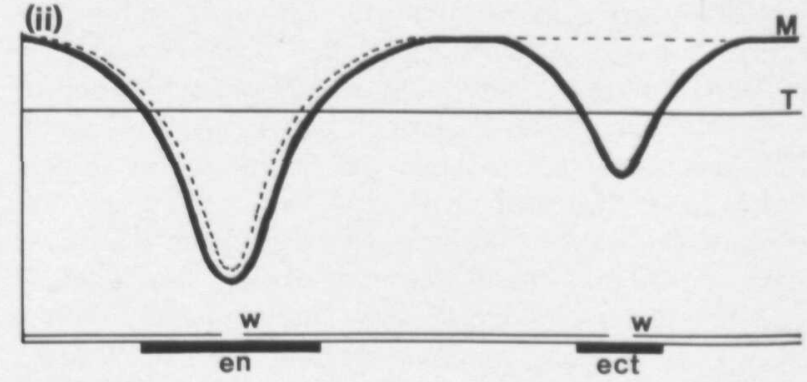

(iii)

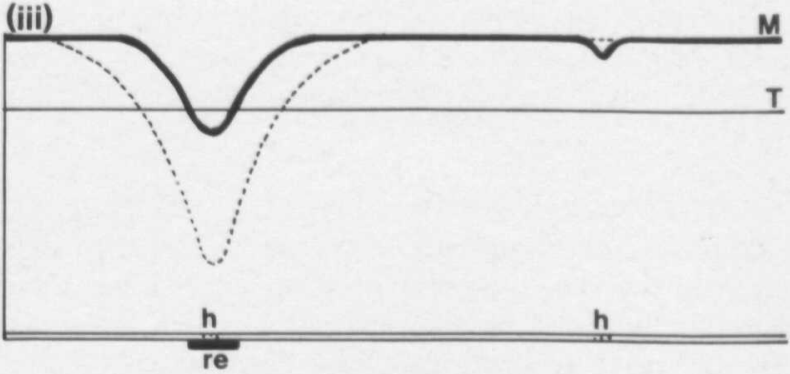

Fig. 6. Morphogen gradient models of eyespot specification. Diagrams show the wing epidermis (ep), the focus (f), wounds caused by cautery (w), the resulting healed area (h), and the extent of the eyespot (bar) specified by the relationship between levels of the morphogen (M) and the response threshold (T). The Bicyclus results indicate that the effect of non-focal cautery must be transient, and that the morphogen gradient must be assessed at a discrete time (rather than continuously, over a protracted period - see Nijhout, 1980). Morphogen and threshold are shown at the time of determination in (i) normal development, and after (ii) late and (iii) early focal and non-focal microcautery (the normal profiles of $\mathrm{M}$ and $\mathrm{T}$ are given by dashed lines). A single threshold is shown, but multiple thresholds are needed for the concentric eyespot pattern. (A) The Source/Threshold model (i) The focus (f) is a local source of M, and the normal eyespot forms where morphogen exceeds threshold $(\mathrm{M}>\mathrm{T})$. (ii) A dip in the profile of $\mathrm{T}$, caused by recent (i.e. late) cautery, results in an ectopic eyespot (ect) where T falls below the basal level of M. A focal cautery also removes the source of $\mathrm{M}$ and hence causes a fall in the gradient, so the eyespot will be enlarged by cautery (en) only if the effect on $\mathrm{T}$ is the predominant rapid response, as shown. (iii) In the extended period after early cautery, the epidermis heals $(\mathrm{h})$, and the transient effects on threshold almost disappear. Due to removal of its source, the morphogen gradient has fallen, greatly reducing eyespot size (re). (B) The Sink model. (i) The focus acts as a sink, destroying $\mathrm{M}$, and the eyespot forms where $\mathrm{M}<\mathrm{T}$. (ii) Transient destruction or loss of $\mathrm{M}$, caused by recent (late) cautery, will induce an ectopic pattern (ect). A focal cautery will cause temporary loss of $\mathrm{M}$ through damage to the epidermis, but will also remove the normal sink, so the eyespot will enlarge (en) only if the overall effect is a decrease in the level of M, as shown. (iii) In the time following an early cautery, the transient effects of damage on $\mathrm{M}$ almost disappear, so no ectopic is formed but, in the absence of the focal sink, the profile of $\mathrm{M}$ has flattened, reducing eyespot size (re).

at the same time on the same wing, indicating that the focus and the late cautery have an additive effect in generating pattern. This is, of course, in direct contrast to the effect of early cautery, which is to eliminate pattern!

After cautery of the large posterior eyespot on the forewing of Precis, Nijhout found eliminations and reductions, but no increases in eyespot size (Fig. 3 of Nijhout, 1980). Even in Bicyclus, enlargements were mainly restricted to the small anterior eyespot, while the posterior pattern usually continued to show decreases in size (Fig.
2C). It seems unlikely, however, that a general difference between anterior and posterior eyespots accounts for the difference in results, as Nijhout (1980) also found that nonfocal forewing cautery did not produce ectopic patterns (although these did occur on the Precis hindwing - Nijhout, 1985a), whereas ectopics readily occur over much of the Bicyclus dorsal forewing (P. M. Brakefield and V. French, unpublished data).

A satisfactory model of eyespot development must explain how cautery can both eliminate and generate the 
pattern, and also the ways in which these responses may differ between species and between their different wing surfaces.

\section{Models of eyespot formation - sources or sinks?}

The effects of cautery on pattern can extend for about 2 $\mathrm{mm}$ on the adult wing, which is equivalent to $0.7 \mathrm{~mm}$ or about 90 cells on the 1 hour pupal wing (whereas cell death extends for less than 10 cells from the site of cautery). The nature of the response thus indicates a long-range mechanism, such as a morphogen gradient (Nijhout, 1980), rather than the short-range interactions which are invoked for most patterning in post-embryonic insect epidermis (French et al, 1976; Martinez-Arias, 1989). The results of cautery and of grafting (Nijhout, 1980; V. French and P. M. Brakefield, unpublished data) indicate that the central focus directs normal eyespot formation. Nijhout (1980) has argued that the focus is the source of a morphogen gradient; the major problem is that this model does not explain how cautery can generate pattern (on the Bicyclus forewing or the Precis hindwing), as it seems implausible that cells respond to death or damage by producing morphogen. There are other ways, however, in which cautery could mimic a focus (Nijhout, 1985a,b), and here we suggest two forms of gradient model which can explain most of the experimental results.

\section{(i) Source/Threshold model}

In a simple source model the focus produces a diffusible morphogen, and an eyespot will form around it, where levels of the resulting gradient exceed a certain threshold. An eyespot pattern would also form elsewhere, however, were cautery to reduce the response threshold below the basal morphogen level (Nijhout, 1985a), as shown in Fig. $6 \mathrm{~A}$. Because cautery can generate a large ectopic eyespot, 'threshold' cannot be cell-autonomous (as in most 'positional information' gradient models, see Wolpert, 1969, 1989), but could be set by the (normally uniform) level of a second diffusible substance (Nijhout, 1985a). If the fall in threshold is transient, an early non-focal cautery will heal and not alter wing pattern (Fig. 6Aiii), whereas a late cautery will induce an ectopic eyespot to form around it (Fig. 6Aii). If focal cautery has the transient effect on threshold, plus a cumulative effect on the morphogen profile through removal of the source, then a late cautery could enlarge the eyespot while an early cautery would reduce or eliminate it (Fig. 6A).

(ii) Sink model

Normal eyespot specification and the effects of cautery can also be understood if both the focus and a wound act as local sinks of morphogen (Nijhout, 1985b,1991), with an eyespot pattern forming where the morphogen falls below threshold level (see Fig. 6B). After early cautery, the epidermis will have healed and temporary damage effects will be gone by the time of determination, leaving no ectopic at the non-focal site, while the cumulative effect of removing the focal sink will reduce or eliminate the normal eyespot (Fig. 6Biii). The damage effect of late cautery will induce an ectopic eyespot and perhaps enlarge a normal eyespot (see Fig. 6Bii).
Both of these models can account in principle for the different responses to cautery seen in Bicyclus (Fig. 2). Grafting experiments show that the small anterior and large posterior dorsal eyespots differ in the strength of their foci (V. French and P. M. Brakefield, unpublished data), and it is plausible that the transient damage effect of late cautery could override loss of the weak anterior focus (giving an enlargement of the eyespot), but not of the strong posterior focus (see Fig. 6). The different response of the ventral pattern (Table 1) may result from lower sensitivity of the ventral epidermis to transient damage, which will reduce or prevent formation of ectopics and eyespot enlargements. The difference between the Precis forewing (Nijhout,1980) and hindwing (Nijhout, 1985a) may also result from different sensitivities to damage, rather than major differences in mechanism, such as the existance of forewing sources but hindwing sinks (see Nijhout, 1991).

One consistent feature of the results remains unexplained, however. Whereas both models predict that eyespot reductions will be most severe after the earliest cautery (as they were in Precis - see Fig. 3 of Nijhout, 1980), the Bicyclus anterior reductions were equally severe at 1 or at 6 hours, and the most severe posterior ones were later, at 6 and 12 hours. The apparent species difference may result from different technique: operations on Bicyclus (but not those on Precis) involved piercing the wing, and this may do less extensive damage to the epidermis (and be less likely completely to ablate the focus) before it separates from the cuticle in apolysis (at 4 hours).

The models rest on many assumptions about the rates and extent of changes following cautery. They explain the results in terms of the removal of a focus (which gradually flattens the morphogen profile) and transient damage (which either effects the morphogen or the response to it). Increasing the severity of cautery would be expected to cause more extensive damage, which would delay healing and hence produce ectopics and enlarged eyespots after an earlier operation. In both species, the severe 6-hour cautery did indeed give ectopics and enlarged eyespots (Table 1), whereas these only occurred later with the mild cautery used in the main experiments. The results of a full series of mild and severe cauteries (V. French and P. M. Brakefield, unpublished) will test these models, and perhaps discriminate between them or suggest a different, more satisfactory model of eyespot formation.

\section{Diffusion gradient models?}

Nijhout $(1978,1980,1985 b)$ has argued that the results of cautery and grafting experiments on Precis are compatible with diffusion of a small polypeptide through the epidermal cells and intervening gap junctions. It appears from the present Bicyclus results that pattern modifications are faster at a lower temperature than in Precis, and so require a much higher morphogen diffusion coefficient. There must remain some doubt over the adequacy of diffusion as a mechanism for establishing a morphogen concentration gradient over a rather large distance in a rather short time (Crick, 1970; see also Bard and French, 1984; Nijhout, 1991).

Nijhout $(1990,1991)$ has extended models of eyespot formation to show that a wide range of the observed wing patterns could be formed by an additive interaction between 
two diffusion gradients generated by morphogen sources (or sinks) at a few standard positions. These models are very impressive, but only further experimental studies can directly demonstrate common developmental mechanisms, such as morphogen gradients, and decide whether diffusion is indeed the means of cell interaction.

We thank the Carnegie Trust and the Universities of Edinburgh and Leiden for financial support, and Els Schlatmann and her helpers for growing maize for very hungry larvae. We are grateful to Fred Nijhout for stimulating exchange of ideas and unpublished results, to Neil Toussaint for once-youthful enthusiasm, and to Jonathan Bard for wit and some wisdom.

\section{References}

Bard, J.B.L. and French, V. (1984). Butterfly wing patterns; how good a determining mechanism is the simple diffusion of a single morphogen? $J$. Embryol. exp. Morphol. 84, 255-274.

Brakefield, P.M. and Reitsma, N. (1991). Phenotypic plasticity, seasonal climate and the population biology of Bicyclus butterflies in Malawi. Ecol. Entomol. 16, 291-303.

Crick, F.H.C. (1970). Diffusion in embryogenesis. Nature 225, 420-422.

French, V., Bryant, P.J. and Bryant, S.V. (1976). Pattern regulation in epimorphic fields. Science 193, 969-981.
Martinez-Arias, A. (1989). A cellular basis for pattern formation in the insect epidermis. Trends in Genetics 5, 262-267.

Nijhout, H.F. (1978). Wing pattern formation in Lepidoptera; a model. $J$. exp. Zool. 206, 119-136.

Nijhout, H.F. (1980). Pattern formation on lepidopteran wings: determination of an eyespot. Devl. Biol. 80, 267-274.

Nijhout, H.F. (1985a). Cautery-induced colour patterns in Precis coenia (Lepidoptera: Nymphalidae). J. Embryol. exp. Morphol. 866, 191-203.

Nijhout, H.F. (1985b). The developmental physiology of colour patterns in Lepidoptera. Adv. Insect Physiol. 18, 181-247.

Nijhout, H.F. (1990). A comprehensive model for colour pattern formation in butterflies. Proc. Roy. Soc. Lond. B. 239, 81-113.

Nijhout, H.F. (1991). The Development And Evolution Of Butterfly Wing Patterns. Washington \& London: Smithsonian Institution Press.

Schwanitsch, B.N. (1924). On the groundplan of wing-pattern in nymphalids and certain other families of rhopalocerous Lepidoptera. Proc. Zool. Soc. Lond. B. 34, 509-528.

Suffert, F. (1927). Zur vergleichende Analyse der Schmetterlingszeichnung. Biol. Zblt. 47, 385-413.

Windig, J. (1992). Quantification of lepidopteran wing patterns using an image analyser. J. Res. Lepid. (in press).

Wolpert, L. (1969). Positional information and the spatial pattern of cellular differentiation. J. theor. Biol. 25, 1-47.

Wolpert, L. (1989). Positional information revisited. Development 107, Suppl. 3-12. 